\title{
Effect of Mulligan Stretching Techniques (TSLR AND BLR) on Biceps Femoris Muscle and Pelvic Rotation by Using Surface EMG and Bubble Inclinometer Respectively
}

Pratishtha, K. \& Jagga, V.

Prem Physiotherapy and Rehabilitation College, Panipat, Haryana.

\section{Abstract}

This study was aimed to investigate and compare the effectiveness of two Mulligan techniques (TSLR and BLR that is Traction Straight Leg Raise and Bend Leg Raise) in improving the biceps femoris muscle performance, flexibility and pelvic rotation. A total of 90 asymptomatic female subjects of 18 to 30 years age were taken which were randomly divided into three groups, that is, two experimental (A and B) and one control group (C). The pelvic rotation ROM, passive straight leg raise ROM and EMG activity were checked in all three groups, after that the BLR, TSLR and Hamstring Self Stretch was provided to Group A, B and C respectively. All the previous readings were checked again. TSLR stretching technique was found to decrease the EMG activity and increase the range of pelvic rotation and passive straight leg raise more than other two groups. Conclusion: Mulligan TSLR stretch is more effective than BLR stretch in improving biceps femoris muscle performance, flexibility and pelvic rotation.

Key Words: Asymptomatic, Passive Straight Leg Raise, Surface EMG, Hamstring

\section{Introduction}

EMG has become a useful tool for the investigation of the muscle activity and hence provides an efficient technique for improving the activity of the muscle. The amount of torque produced by a muscle depends on the number of motor units activated, the muscle length, and the moment arm of the muscle. These variables have been studied in various combinations, but there is lacks of studies that have investigated the relationship among EMG activity, and muscle length before and after stretching as stretching is also a muscle lengthening procedure. During maximal isometric contraction, an increase in integrated Electromyographic activity and a decrease in torque occurrs as the muscle is shortened, the opposite occurrs when the muscle is in lengthened positions. A greater difference in this relation is noted when the respective EMG activity and torque are held constant (Lunnen et al, 1981).

Mulligan bent leg raise (BLR) technique has been described as a means of improving range of straight leg raise(SLR) in subjects with LBP and/or referred thigh pain (Hall et al, 2006b). Mulligan has also described the traction straight leg raise (TSLR), which is said to improve the range of straight leg raise (SLR) in patients. Furthermore, it has been suggested that improving the range of SLR has a beneficial effect in restoring normal movement and reducing the degree of impairment due to low back dysfunction (Hall et al, 2006a). The present study has provided useful information about the internal as well as the visible changes in the muscle due to 
the application of the mulligan (TSLR and BLR) techniques thus providing a better approach for the treatment. The purpose of the study was to establish the effect of TSLR and BLR mulligan technique on the biceps femoris muscle performance, flexibility and pelvic rotation.

\section{Materials \& Methods}

90 asymptomatic female subjects were randomly assigned to each Group A (BLR Experimental group), Group B (TSLR Experimental group), and to Group C (Control group) using convenience random sampling method (30 in each group). The informed consents of subjects who were able to read and write simple English were collected with prior permission. Inclusion criteria included age of 18 to 30 years and female gender having positive passive knee extension test. The subjects were excluded on the basis of any previous medical history of lower limb injury or pain from last one year, previous fractures or surgery to back, pelvis, hip and knee, any pathological or inflammatory condition that could affect motion, pregnancy, general medical conditions like hypertension, diabetes and any neurological conditions. The pelvic rotation was checked first, by using bubble inclinometer during forward bending followed by hamstring flexibility using the PSLR test, a standard test shown to have a reliability of 0.97 . The EMG (by the Neuroperfect 2 channel EMG NCV EP instrument) was then checked on biceps femoris muscle during the maximum isometric contraction produced against resistance of the repetition maximum of the subject on the DeLorme table. Group A, Group B, and Group C received three BLR, TSLR and hamstring self stretch respectively. After the completion of the stretching procedure, all pre-test readings of EMG, PSLR, and pelvic rotation were again recorded.

\section{Results \& Discussion}

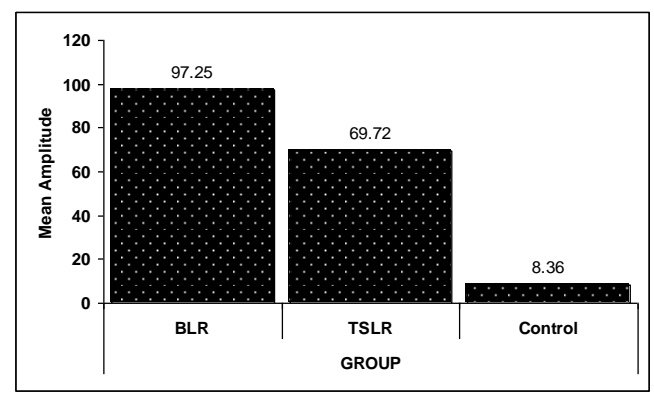

Figure 1: Comparison of change in EMG activity among the three groups

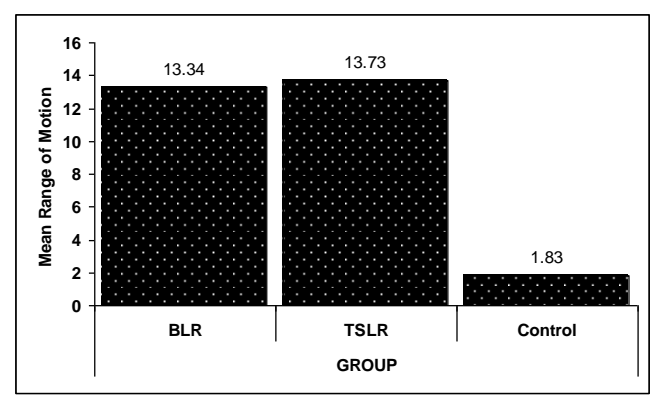

Figure 2: Comparision of change in PSLR motion among the three groups

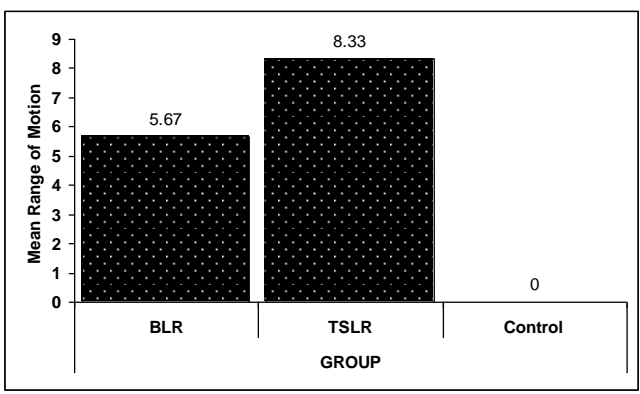

Figure 3: Comparision of change in Pelvic Rotation ROM among the three groups

Results were analyzed by using one way anova and paired t-test by using SPSS version 15 . The within group analysis of the EMG showed that in Group A (BLR) there was an increase in EMG activity with a percentage of $22.52 \%$ and in Group 
B (TSLR) there was a decrease in EMG activity by $14.64 \%$, but no significant difference in Group C (Control) was observed. The PSLR within group analysis showed a significant difference between the pre and post readings in both groups. Group A (BLR) with an overall improvement of $23.68 \%$ and in Group B (TSLR) improvement is of $25.69 \%$ and in Group C (Control) that is of $3.29 \%$. The within group analysis of the Pelvic Rotation also showed that in Group A (BLR) there was an overall improvement in range of pelvic rotation that is of $14.20 \%$ and of $18.20 \%$ in Group B (TSLR) but no difference in Group C (Control).

This study investigated the effectiveness of the two Mulligan techniques that is Traction Straight Leg Raise (TSLR) and Bend Leg Raise (BLR) in improving biceps femoris muscle performance, flexibility and pelvic rotation. The results of this study reveal that the TSLR (Traction Straight Leg Raise) stretch is more effective in improving muscle activity, flexibility and pelvic rotation than the BLR (Bend Leg Raise) and hamstring self stretch.

In the TSLR group the EMG activity decreased by $22.52 \%$, thus suggesting improved muscle performance as decrease in muscle activity shows that muscle is able to do the same work with less recruitment of motor units thus rendering it more efficient, while in the BLR group the EMG activity increased by $14.64 \%$. A study done by Lunnen et al (1981) also support these results who found that changes in the moment arm of a muscle appear to affect the production of EMG activity differently. During maximal isometric contraction, an increase in integrated electromyographic activity and a decrease in torque occurred as the muscle was shortened; the opposite occurred when the muscle was in lengthened positions. The TSLR group also showed a relatively greater increase in the straight leg raise ROM (25.69\%) than the BLR group (23.68\%). Thus it is in agreement to the results of the study done by Lunnen et al (1981). Further the increase in the muscle length resulted in a decrease in the EMG activity thus rendering a muscle more efficient. There is also increase in the PSLR range in the BLR group, but alongwith it, the EMG activity has also been observed to increase. These observations are in agreement with the study done by Hideaki et al, 2002). Hideaki \& coworkers found that the EMG activity of hamstring muscles during maximum isometric knee flexion varies with change in muscle length or joint angle. In this study the EMG activity has increased because of knee flexion, so this may be the reason of increase in the muscle activity in the BLR group as in the BLR stretch, the knee was in flexion thus leading to an increase in muscle activity.

The results of this study differ from the results of the study done by Andersen et al (2010) who reported that comparable high levels of muscle activation were obtained during resistance exercises with dumbbells and elastic tubing. In the present study the EMG activity was checked during the maximal resistance given by the DeLorme weights, but the EMG activity decreased in the TSLR group. The TSLR group also showed the increase in the straight leg raise range and pelvic rotation which are in accordance with the study done by Bellew et al (2010) who showed a positive correlation 
between hamstring flexibility and pelvic rotation. It was also observed that total forward bending range is similarly influenced by hamstring flexibility. The increase in straight leg raise range and a decrease in the EMG activity in TSLR group may be due to the fact that during TSLR stretch, various receptors exert an inhibitory influence on lower limb alphamotoneuron activity. Golgi tendon organs around the knee, hip and spine probably initiate various segmental reflex pathways during traction of the limb. Likewise, Golgi tendon organs are activated during large amplitude stretching movements such as SLR. This processing of information in the nervous system may inhibit the activity of the muscles being lengthened during SLR by dampening the afferent activity of type II muscle spindles or by decreasing motor neuron excitability via 1-b fibers. Hence, improvement in range of SLR may be directly related to inhibition of the hamstring muscles rather than changes due to stretch tolerance.

\section{Conclusion}

Based on the results of the present study, it is concluded that TSLR Mulligan stretch is better than BLR Mulligan stretch in improving the biceps femoris muscle performance, flexibility and pelvic rotation.

\section{References}

Andersen, L.L., Andersen, C.H., Mortensen, O.S., Poulsen, O.M., Bjørnlund, I.B.T. and Zebis, M.K. 2010. Muscle Activation and Perceived Loading During Rehabilitation Exercises: Comparison of Dumbbells and Elastic Resistance. Phys. Therapy, 90: 538549.

Bellew, S., Ford, H. and Emma Shere, E. 2010. The Relationship between Hamstring Flexibility and Pelvic Rotation around the Hip during Forward Bending. The Plymouth Student Journal of Health \& Social Work, 2:19-29.

Fauth, M.L., Petushek, E.J., Feldmann, C.R. et al. 2010. Reliability of Surface Electromyography during Maximal Voluntary Isometric Contractions, Jump Landings, and Cutting. J. Streng. \& . Res., 24(4): 1131-1137.

Hall, T., Beyerlein, C., Ulla Hansson, U., Lim, H.T., Odermark, M., Sainsbury, D. 2006a. Mulligan Traction Straight Leg Raise: A Pilot Study to Investigate Effects on Range of Motion in Patients with Low Back Pain. The J. Man. \& Manip. Ther., 14(2): $95-100$.

Hall, T., Hardt, S., Axel Scha, A., Lena Wallin, L. F. 2006b. Mulligan bent leg raise technique - a preliminary randomized trial of immediate effects after a single intervention. Manual Therapy, 11(2): 130-135.

Hideaki, O., Ryo, Y., · Oyama, M., Akasaka, K. Ihashi, K., Yasunobu, H. 2002. EMG-angle relationship of the hamstring muscles during maximum knee flexion. J. EMG Kinesiol., 12(5): 399-406.

Lunnen, J.D., Yack, J., LeVeau, B.F. 1981. Relationship Between Muscle Length, Muscle Activity, and Torque of the Hamstring Muscles Phys. Ther., 61:190-195.

Marek, S.M., Cramer, J.T., A. Fincher, L., Massey, L.L., Dangelmaier, S.M., Purkayastha, S., Kristi, A Fitz, K.A., and Culbertson, J.Y. 2005. Acute Effects of Static and Proprioceptive Neuromuscular Facilitation Stretching on Muscle Strength and Power Output. J. Athl. Train., 40(2): 94-103.

O'Sullivan, K. Smith, S.M. and Sainsbury. D. 2010. Electromyographic analysis of the three subdivisions of gluteus medius during weight-bearing exercises. Sports Med Arthrosc. Rehabil. Ther. Technol., 2: 17.

Roseborrough, A., Lebec, M. 2007. Differences in static scapular position between rock climbers and a non-rock climber population. North Am. J, Sports Phy, Therap., 2(1): 4450.

Sihvonen, T. 1997. Flexion Relaxation of the Hamstring Muscles during Lumbar-Pelvic Rhythm. Archv. Med. Rehab., 78(5): 486490.

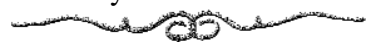

